\title{
SCALABLE OBJECT DETECTION, TRACKING AND PATTERN RECOGNITION MODEL USING EDGE COMPUTING
}

\author{
Dipak Pudasaini \\ Abdolreza Abhari \\ Department of Computer Science \\ Ryerson University \\ 350 Victoria Street, Toronto, ON, Canada \\ \{dpudasaini,aabhari\}@ryerson.ca
}

\begin{abstract}
The Internet of Things (IoT) devices such as sensors and video cameras have small memories and less computational power. The video analytics of traditional approaches for detection, tracking and pattern recognition of moving objects used only in the cloud. This approach suffered from high latency and more network bandwidth to transfer data into the cloud. We address this problem by using edge computing devices between IoT devices and the cloud. We propose a new framework for scalable object detection, tracking and pattern recognition of moving objects that relies on dimensionality reduction with edge computing architecture. We also propose a scalable object detection and tracking method based on You Only Look Once (YOLO) method. The experiment demonstrates that our proposed method will save network bandwidth and processing time. The performance of object detection and tracking model is greater than $96 \%$. This shows that our method has greater performance than existing models.
\end{abstract}

Keywords: object tracking, pattern recognition, edge computing, dimensionality reduction, YOLO.

\section{INTRODUCTION}

Devices in wireless communication technology have been increased dramatically in recent years. Internet of Things (IoT) devices such as video cameras and sensors are very common for many applications. It has been forecasted that more than 25 billion devices will be connected to the internet in 2020 (Mahdavinejad et al. 2018). IoT devices have small size memory and limited computing power. The main IoT device for video analytic application is video cameras. Video analytics from video cameras are used for traffic control, surveillance and digital assistance for real time decisions. The traditional video analytic system used only the cloud based approach for object detection and classification (Anjum et al. 2016).

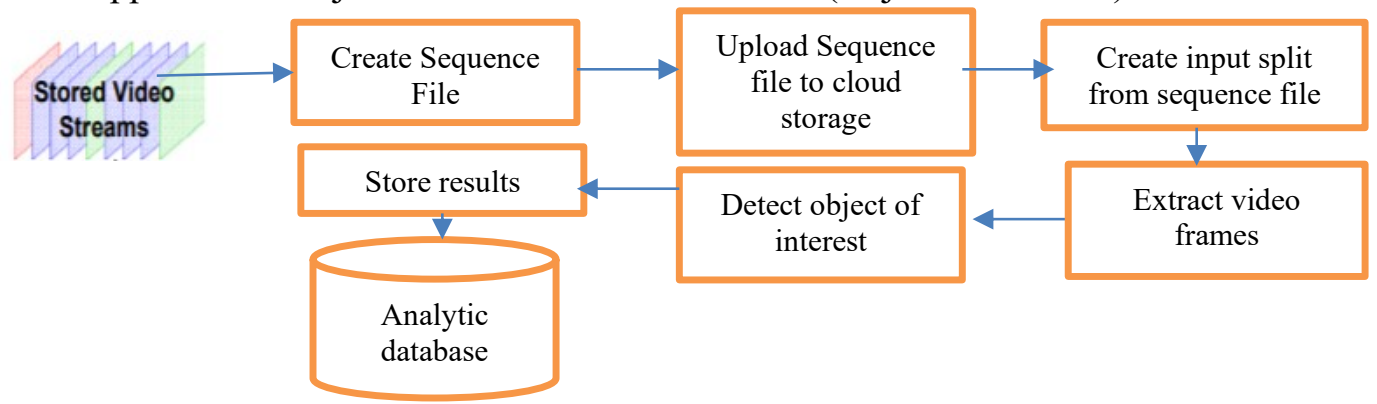

Figure 1: Video stream analysis in the cloud (Anjum et al. 2016). 
In traditional video analytic systems, video data is transferred to the cloud where video frames are extracted, and objects are detected and analyzed. The traditional model has suffered from high latency and more network bandwidth to transfer data from IoT devices to the cloud. The cloud-based approach is the centralized approach in which the video analytic is taken place.

We proposed a model using edge computing architecture to minimize the problems of using only cloudbased architecture for detecting, tracking and pattern recognition of moving objects in the video files or real time videos. Edge computing is an open platform to store and process data at the edge of the network. Edge computing allows any application to be processed between the cloud and the users. It is a distributed computing approach that allows data to be processed and analyzed in real time near the data collection source (Khan et al. 2019). In this approach, the object detection and tracking are performed in the edge devices, and then pattern recognition is performed in the cloud device. In the proposed approach, data does not need to be uploaded directly to the cloud or to a centralized data processing system. The input video data is converted into text data in edge devices, and then is transferred into the cloud device for pattern recognition. Therefore, the proposed edge computing mechanism will result in less traffic in the internet given the fact that images and videos make a substantial chunk of internet bandwidth.

There are different types of object tracking methods such as color based, motion-based, shape-based and more (Yilmaz, Javed and Shah 20006). The more popular object detection methods are Recurrent Convolutional Network (R-CNN), Fast R-CNN, Faster R-CNN and You Only Look Once (YOLO). In our proposed approach, the object detection and tracking will be done in the edge of the network. The YOLO method is more popular because of its accuracy and fast performance. Therefore, we use YOLO as a base method for object detection. Moreover, the YOLO method is combined with the centroid tracking to detect and track the moving objects.

The rest of the paper is organized as follows: Section 2 presents problem statements and contributions. Section 3 presents the literature review of the proposed work. Section 4 presents the details of our proposed approach. The experimental results are explained in Section 5 and Section 6 summarizes the conclusion of this paper.

\section{PROBLEM STATEMETS AND CONTRIBUTIONS}

In this paper, we will propose a framework for scalable detection, tracking and pattern recognition of moving objects that relies on edge computing architecture. The novel part of addressing the problem is using a method that relies on dimensionality reduction with edge computing architecture to minimize processing time and network bandwidth.

Let the video analytic problem be

$$
\mathrm{P}=\left\{\mathrm{s}_{1}, \mathrm{~s}_{2}, \mathrm{~s}_{3}, \ldots, \mathrm{s}_{\mathrm{n}}\right\} .
$$

Where, $s \in p$ is the different parts (i.e., each stage) of the video analytic problem and $\mathrm{P}$ is the set of all the stages. For example, for object tracking and pattern recognition, $\mathrm{s}_{1}$ is motion detection, $\mathrm{s}_{2}$ is object detection, $\mathrm{s}_{3}$ is object tracking and trajectory extraction, $\mathrm{s}_{4}$ is trajectory pattern recognition.

Let the dimensionality of original data during each stage be $d_{i} \in \mathrm{D}$ :

$$
\mathrm{D}=\left(\mathrm{d}_{1}, \mathrm{~d}_{2}, \mathrm{~d}_{3}, \ldots, \mathrm{d}_{\mathrm{n}}\right) \text {. }
$$

Where, $\mathrm{d}_{\mathrm{i}}$ is the $\mathrm{i}^{\text {th }}$ dimension of original data.

The problem is to find a framework called function frelated to each process $s \in p$ that reduces the dimension $\mathrm{d}_{\mathrm{i}}$ of original data.

$$
\mathrm{f}: \mathrm{D} \rightarrow \mathrm{N}^{+} \text {. }
$$


Where, $\mathrm{N}^{+} \in \mathrm{D}$ is the resulted dimension where the result of each stage has lower dimensionality than the previous stage.

Our main contributions are to:

- Design a framework for scalable object detection, tracking and pattern recognition of moving objects that relies on dimensionality reduction with edge computing architecture.

- Design a scalable object detection and tracking method using YOLO as a main method for moving objects.

- Design an experiment to verify scalable object detection, tracking and pattern recognition of moving objects that relies on dimensionality reduction with edge computing architecture.

- Design an experiment to verify the efficiency of object detection and tracking method.

\section{LITERATURE REVIEW}

Edge computing focuses to bring services and utilities of cloud computing closer to the user for fast processing of data intensive applications (Khan et al. 2019). The edge computing approach is used to offload computing application, data and services from the cloud computing to the edge of the network (Shi et al. 2016). The emerging technologies in cloud computing ensure to deliver cloud services for mobile computing, scalability and privacy policy enforcement for the Internet of Things (IoT), and the ability to mask transient cloud outages (Satyanarayanan 2017). The IoT devices spread all around the world to input and automate the processing of data (Yadav and Prasad 2019). The real time video analytics for traffic monitoring system has been proposed (Ananthanarayanan et al. 2017). The cost-effective technique for aerial surveillance in which large computation tasks are in the cloud and limited computation task in Unmanned Aerial Vehicle (UAV) device using an edge computing technique has been proposed (Alam et al. 2019).

In (Murshed et al. 2019), the survey of machine learning algorithms in the network edge has been presented. The transmission of raw data to the cloud causes many problems such as increased communication costs, delayed system response and privacy concerns. If the additional computing devices at the edge of a network closer to IoT devices are placed, they will be used to run machine learning algorithms. By this technique, the machine learning is deployed at the edge of computer networks. The edge-based video analytics system uses deep learning to recognize the objects in a large scale IoT video stream has been proposed (Ali et al. 2016). The three stages of this model that are frame loading, preprocessing and object detection are performed in edge infrastructure, and then object recognition is performed in the cloud.

There are variety of methods for detection and tracking of moving objects. The scalable pattern recognition and real time tracking of moving objects has been proposed (Pudasaini and Abhari 2019). The OpenCV and image processing techniques have used to develop a tracking model of moving objects. The real-time data mining of the sequence of frames for processing trajectories, collected by a security camera has been explained (Abhari, Banihashemi and Li 2018). The color-based object detection and tracking method using simple tools and techniques of computer vision have been proposed (Nidhi 2015).

There are various types of object tracking and classification techniques using a neural network. Convolutional Neural Network (CNN) has been extensively used for pattern recognition and image recognition for moving objects in videos. The real time object recognition model that uses deep CNN to extract deep features has been proposed (Yang, Wang and $\mathrm{Wu} 2018$ ). The detection of fast-moving vehicles that uses CNN has been proposed (Zhu 2017). A multi-level three-dimensional convolutional neural network for moving objects recognition has been described (He, Mao and Yi 2015). The real time object detection and tracking that use deep learning and OpenCV has been proposed (Chandan et al. 2018). This method has used Single Shoot Detector (SSD) for detection and tracking of moving objects. 
Centroid based tracking of moving objects has been proposed (Singh 2017). In this method, centroid based boundary detection algorithm has proposed to find the centroid of moving objects. An algorithm for tracking the moving objects in a real time video based on background subtraction has been presented (Naha et al. 2014). The centroid point of each frame is stored in the form of vector to identify the path of moving objects. Software Defined Networking (SDN) is a set of networking principles whose goal is to create a flexible, dynamic, and scalable network through software-based management and configuration (Farhady, Lee and Nakao 2015). SDN is the latest network architecture where network control is decoupled from forwarding and directly controlled by programmable units (Xia et al. 2015). It is more popular than traditional networking approach by two characteristics, one decoupling from data and control plane, another programmability on the control plane.

The existing frameworks for moving object detection, tracking, and pattern recognition are limited to a few number of moving objects and does not provide dimensionality reduction. Our approach provides a framework for dimensionality reduction and it is scalable for detection, tracking and pattern recognition of moving objects that rely on edge computing architecture.

\section{PROPOSED METHOD}

We proposed a system to provide scalability and dimensionality reduction for object detection, tracking and pattern recognition that relies on edge computing architecture. Figure 2 explains the proposed architecture that uses edge computing. The video camera captures the motion of the vehicles which will be stored in the local servers. There are numbers of local servers in different places to store the motion of the vehicles. These local servers take the advantage of edge computing in performing real time object tracking (which require processing power) on edge servers to make the process scalable. By extracting trajectories and sending them to the cloud, the pattern recognition of the trajectories that came from different edge servers is carried out with machine learning algorithms in cloud computing system.

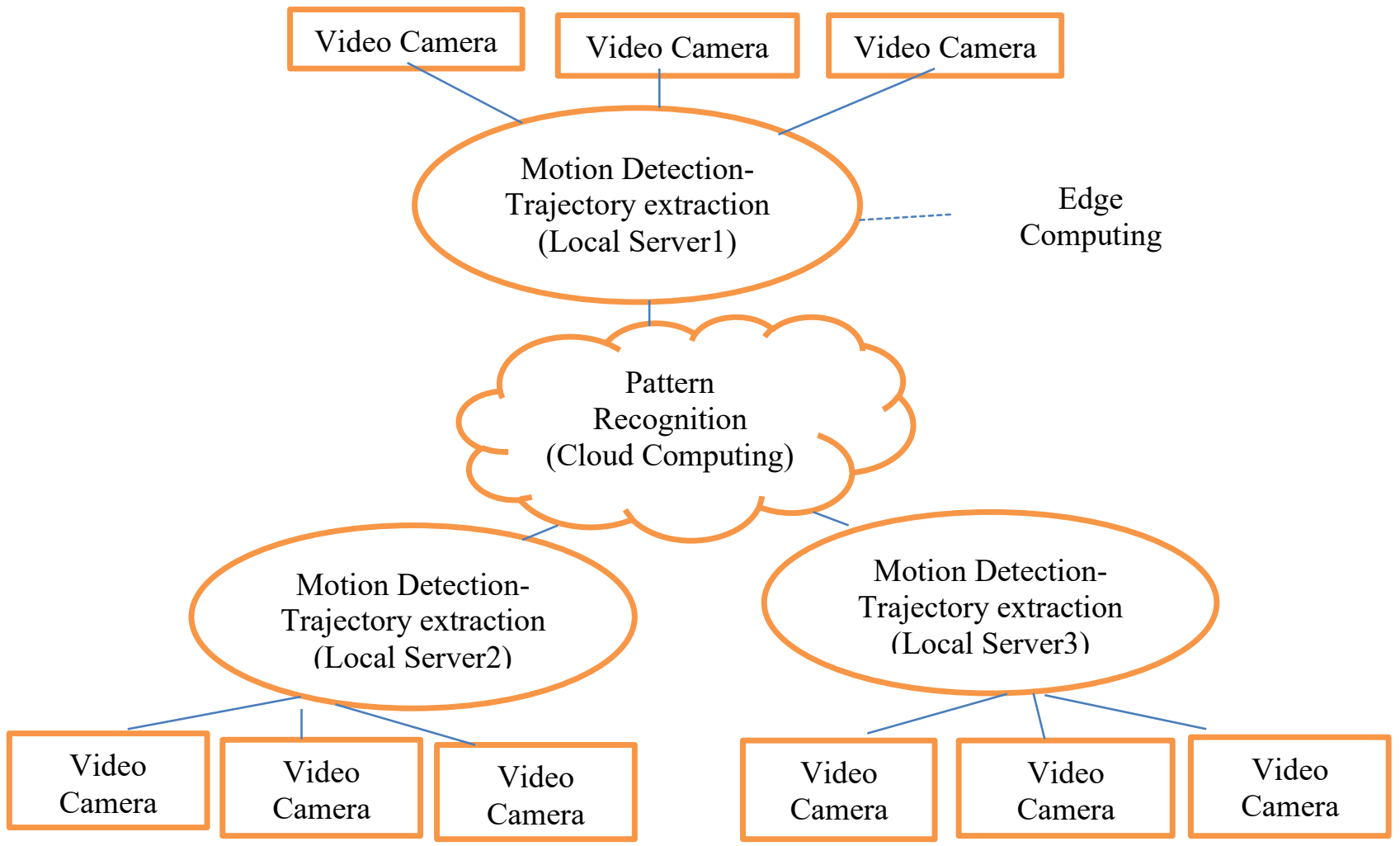

Figure 2: System architecture for dimensionality reduction and Scalability test using edge computing. 
We have combined different programs of a whole video analytic application to be able to do dimension reduction in the application level (Not inside program). We used YOLO and centroid tracking method in the edge level to process the large dimension of data (i e video frames), and then passed the dimensionality reduced data to the cloud level. The lower dimensional data (i e trajectory in text format) is processed in the cloud. This framework measures the network performance using edge computing with cloud and only cloud and also saves bandwidth by extracting trajectories using edge computing technique and by directly passing video files to the cloud. It also measures the processing time using edge computing with cloud and only cloud.

Software Defined Networking (SDN) is the better technique to implement this framework. The video data is entered from the video source to the edge network to the central cloud by using Software Defined Network (SDN). SDN is a paradigm that provides the flexibility to deploy custom work flows on hardware by programming the control plane.

The system architecture of our proposed model consists of three main components: Capturing images from video camera, Motion detection and trajectory extraction, and Pattern recognition.

\subsection{Capturing Images from Video Camera}

The number of IoT devices are increasing day by day. The main IoT devices used for video analytic system is video camera. It is only used to capture the real time image and send to the proper place. In our proposed model a video camera captures the images and sends them to the local server near to the video camera. The images might be read from video files that are from any memory location.

\subsection{Motion Detection and Trajectory Extraction}

The object detection and tracking are done in the edge of a network as shown in figure 3. There are different types of popular methods for object detection and tracking. The YOLO method is more popular because of its fast performance. We use YOLO as a base method for object detection. We will find the centroid of each object in each frame to track the moving objects. The YOLO method is combined with the centroid tracking method to detect and track the moving objects. Finally, the trajectories are extracted which are passed to the cloud for pattern recognition. The overall process is explained in figure 3 .

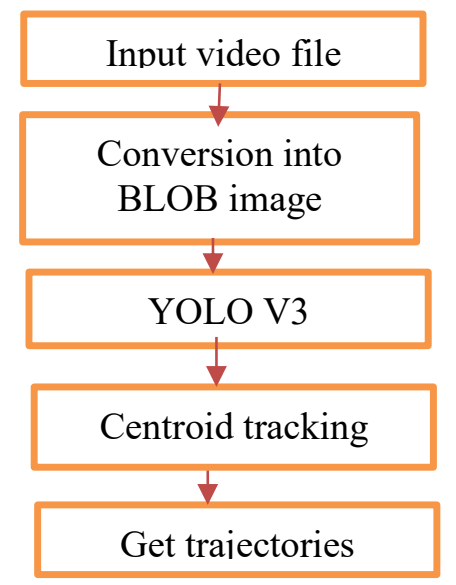

Figure 3: Motion detection and trajectories extraction.

\subsubsection{Input Video File}

Videos are a sequence of frames over time. We read a video frame by frame. For each frame, we apply our tracking algorithm. 


\subsubsection{Conversion into BLOB Image}

We take each frame and form the BLOB (Binary Large Object) of each frame to pass them on YOLO model. BLOB images are the popular format to transfer data over an internet. OpenCV is used to get BLOB from frame images, which creates 4-D BLOB from images. Applying a BLOB helps to increase the performance of detection. Within the same concept of BLOB, we can resize, scale and crop the image without losing information. YOLO model accepts the image with a shape factor of 32 . So a BLOB of small size increases the speed with lower accuracy.

\subsubsection{YOLO V3}

YOLO (You Only Look Once) is the object detection/localization algorithm. YOLO weights and configures files trained on the COCO dataset is used to find the bounding box of 80 objects. YOLO takes the BLOB image and gives object's bounding box, its class id, and the accuracy for that class (Redmon et al. 2016). When the image is input in the form of pixels then it passes through the neural network similar to CNN, then the vector of bounding boxes and class predictions are in the form of output. The input image is divided into an $S \times S$ grid of cells. Each grid cell predicts $B$ bounding boxes as well as $C$ class probabilities. The bounding box prediction has 5 components: $(\mathrm{x}, \mathrm{y}, \mathrm{w}, \mathrm{h}$, confidence), where $(\mathrm{x}, \mathrm{y})$ coordinate represents the center of the bounding box, $(\mathrm{w}, \mathrm{h})$ represents width and height of the bounding box. The confidence score is the score of predicting the object in a box. For feature extraction, it is passed to CNN with 24 convolutional layers followed by 2 fully-connected layers.

\subsubsection{Centroid Tracking}

Centroid tracking works on the idea of finding the centroid of objects using a bounding box, and then checking its distance with the centroid of other objects on another a frame. The distance between previous centroids and current centroids are calculated using Euclidian distance. Then centroid tracking system chooses the minimum distance among them. The object is tracked by assigning an object id on each of the detected objects.

\subsubsection{Get Trajectories}

After using the centroid tracking method, we can get the trajectories of each moving object. The trajectory path is the set of two-dimensional points $(\mathrm{x} 1, \mathrm{y} 1),(\mathrm{x} 2, \mathrm{y} 2),(\mathrm{x} 3, \mathrm{y} 3), \ldots,(\mathrm{xn}, \mathrm{yn})$. The image data is converted into text data so that the dimensionality of the video file is extremely reduced.

\subsection{Pattern Recognition}

When the video data are processed in edge computing devices, the dimensionality of the data is extremely reduced. The image data is converted into text data. We get the trajectories of the motion of the moving objects in text formats. Then this data will be passed into the cloud for pattern recognition. We will use machine learning algorithms to find the pattern of the moving objects in the cloud.

\section{EXPERIMENTS AND RESULTS}

The object detection, tracking and trajectory extraction of our proposed model was implemented in a machine with Windows 10 operating system. It was implemented and tested on Intel Core I5 CPU @ $2.3 \mathrm{GHz}$ with $8 \mathrm{~GB}$ RAM. The programming platform for detection and tracking the objects was Python 3.6 and the OpenCV library. The Python with anaconda environment was used for its implementation. 


\subsection{Overall System Performance}

There are a number of network simulators to measure the performance of this type of system. DEVS (Discrete Event System Simulation) is a flexible modeling and simulation method that is more popular for user mobility and handover process (Kazi, Wainer and Da Silva 2017). GNS3 is an open source network simulator that allows for the simulation of complex networking structure which is used to implement the real networking scenarios (Ahmed, Saleem and Waseem 2015). iFogSim is the network simulator that can be used to model IoT devices with edge devices and the cloud (Gupta et al. 2017). The iFogSim is useful for creating edge and cloud devices to simulate the system. The overall performance of our proposed model was measured by using the iFogSim simulator. We have four stages in the video analytic system: motion detection (s1), object detection (s2), object tracking and trajectory extraction (s3), and trajectory pattern recognition (s4).

Motion detection (s1): The video camera continuously captures the raw video stream. Then the detection of motion forwarded to an object detection module.

Object detection (s2): The object detection module receives video streams from the motion detection module. This module extracts features of the objects to detect them. The video frame may consist of more than one object. The individual objects are detected in this module.

Object tracking and trajectory extraction (s3): The object tracking module receives the results from currently detected objects. Then, the object tracking module tracks the path of moving objects to extract their trajectories.

Trajectory pattern recognition (s4). The object tracking module tracks the path of moving objects and then forwarded it to the pattern recognition module to find the pattern of these objects.

In this experiment our main objective is to reduce the dimensionality of data and improve the network performance and execution time of the overall system using edge network. Here a prototype of the system is implemented using an edge network with the cloud and only the cloud. There are number of cameras in different places in which frames are detected. We have two scenarios: one is the detected motion (s1) directly goes to the cloud for object detection (s2), object tracking and trajectory extraction (s3), and trajectory pattern recognition (s4). Another is detected motion (s1) goes to edge devices for object detection (s2), and object tracking and trajectory extraction (s3), then goes to the cloud for trajectory pattern recognition (s4).

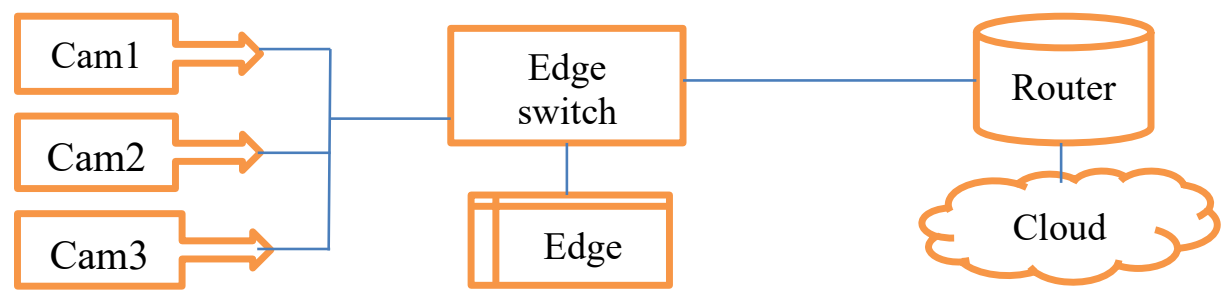

Figure 4: Experimental set up for object tracking and pattern recognition.

The experimental setup is created using iFogSim simulator. The experimental setup consists of video cameras, edge devices, cloud and gateways (routers, switches). Figure 4 explains the experimental setup of our proposed model. Cam1, Cam2 and Cam 3 are video cameras which capture the video stream to find the motion of an object. Switch moves data from video sources to edge devices for object detection and tracking. Router moves the processed data of edges into the cloud.

In this experiment, there are 3 places to keep video cameras where each place contains 3 video cameras. The parameters setting in the cloud device are: MIPS $=22400, \mathrm{RAM}=20000$, Up bandwidth $=50$, Down bandwidth $=5000$, level $=0$, Rate per MIPS $=0.01$, Busy power $=16^{*} 103$, Idle $=16^{*} 83.25$. The parameters 
setting in the edge devices are: MIPS=2500, RAM=3500, Up bandwidth=9000, Down bandwidth=9000, level=1, Rate per MIPS=0.0, Busy power=107.3, Idle=83.4.

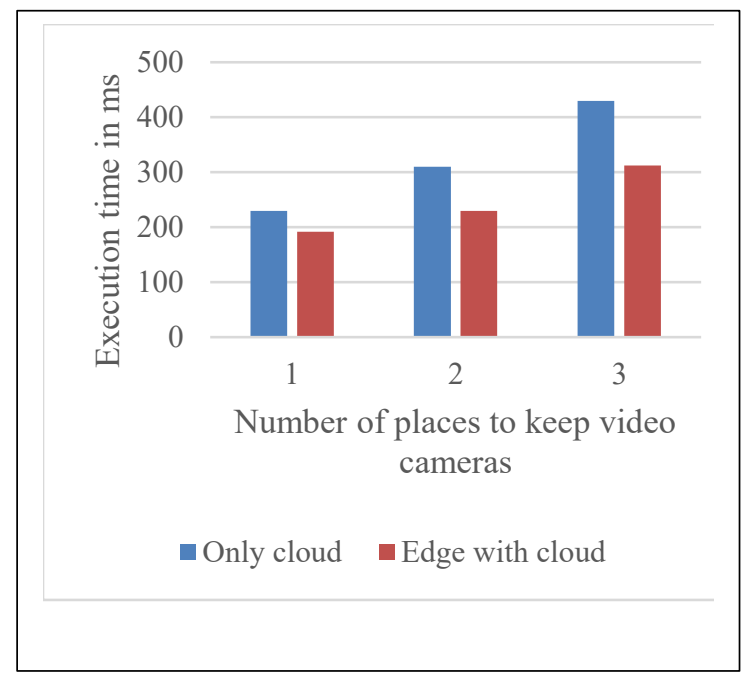

Figure 5: Comparison of execution time between edge with cloud and only the cloud.

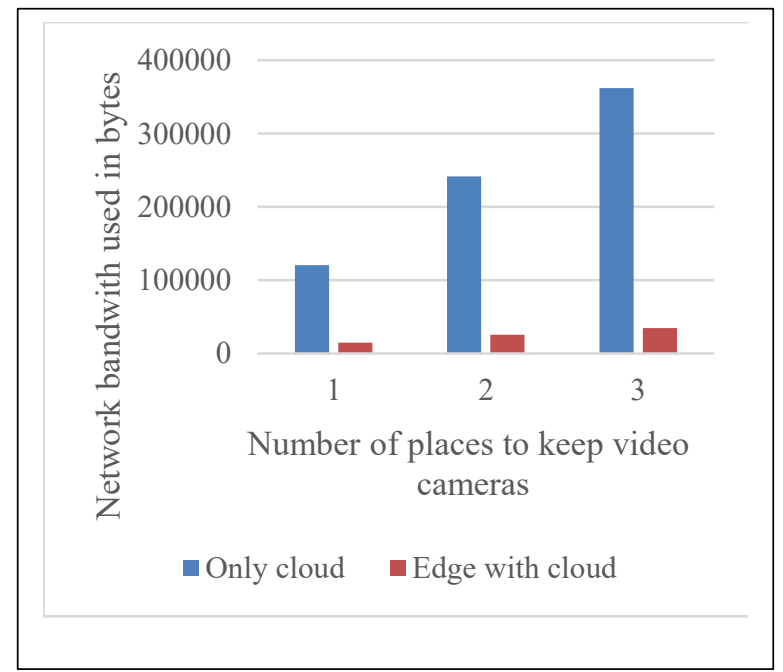

Figure 6: Comparison of bandwidth between edge with cloud and only the cloud.

Figure 5 and 6 demonstrates the comparison of performance of our proposed system (edge networking with cloud) and only the cloud architecture. The network bandwidth and execution time are reduced by using edge network with cloud. If we passed video data directly to the cloud, more network bandwidth will be needed. But if the video data are processed in the edge device, the dimensionality of data is reduced and only the text data are passed to the cloud for pattern recognition. This framework reduces network bandwidth and execution time which is described in figure 5 and 6 . This shows the performance of our proposed model.

\subsection{Object Detection and Tracking Performance}

The object detection and tracking is done in the edge device of our proposed framework. Object detection and tracking system can use any types of videos from social media or stored in database, we can also create new videos. In this experiment, the YouTube videos were collected and tracked in real time to test this system. Most of the videos for testing the system were real videos from social networking sites. We prepared some videos in a real street and parking lots where vehicles are moving. The online tracking is shown in Figure 7 to Figure 10. Each figure shows the moving objects at a given time and rectangle around objects shows tracking of their motion.

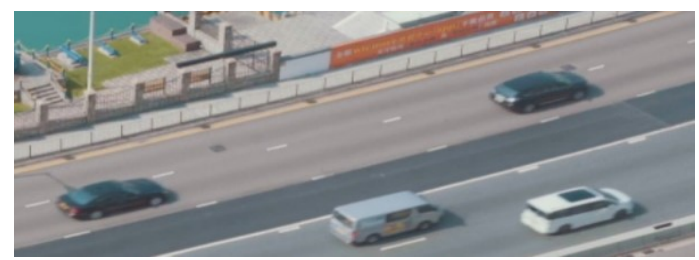

Figure 7: Sample 1 video.

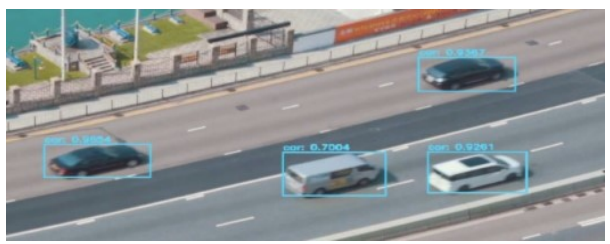

Figure 8: Tracking of sample 1 video. 


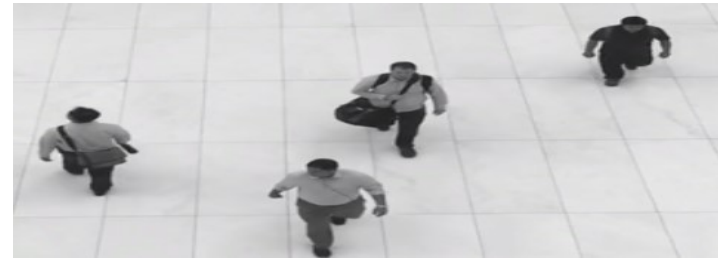

Figure 9: Sample 2 video.

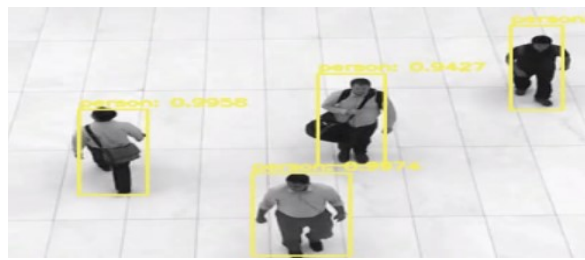

Figure 10: Tracking of sample 2 video.

By using experimental study, we verify our tracking model by finding tracking rate, recall and precision. Table 1 presents the True Positive (TP), False Positive (FP) and False Negative (FN) values of standard real time videos and finds the recall, precision and tracking rate (Gokul, Santosh Kumar and Shreeraj 2012).

$\mathrm{TP}=$ Tracked the actual object

$\mathrm{FP}=$ Tracked the object that do not correspond to actual object

$\mathrm{FN}=$ Object not tracked

Tracking rate $=(\mathrm{TP} * 100) /(\mathrm{TP}+\mathrm{FN})$

Recall $=\mathrm{TP} /(\mathrm{TP}+\mathrm{FN})$

Precision $=\mathrm{TP} /(\mathrm{TP}+\mathrm{FP})$

Table 1: Recall, precision and tracking rate of different videos.

\begin{tabular}{|c|c|c|c|c|c|c|}
\hline Based on objects in videos & TP & FP & FN & Recall & Precision & $\begin{array}{c}\text { Tracking } \\
\text { Rate }\end{array}$ \\
\hline Number of Vehicles only & 400 & 7 & 8 & 0.98 & 0.98 & 98 \\
\hline $\begin{array}{c}\text { Number of Pedestrian and vehicles } \\
\begin{array}{c}\text { Number of Vehicles, pedestrians } \\
\text { and others }\end{array}\end{array}$ & 245 & 6 & 8 & 0.97 & 0.98 & 97 \\
\hline
\end{tabular}

From table 1, we found that the proposed tracking model has good performance because tracking rate is more than $96 \%$. The tracking has very good performance than other methods.

The execution time of this model is faster than other existing models. The average execution time per frame is 1.5 seconds. This shows the execution time per frame is faster for detection and tracking of moving objects. When our object tracking algorithm is run in the local server, the movement path of an object in the form of two-dimensional points $(\mathrm{x} 1, \mathrm{y} 1),(\mathrm{x} 2, \mathrm{y} 2),(\mathrm{x} 3, \mathrm{y} 3), \ldots,(\mathrm{xn}, \mathrm{yn})$ will be extracted. The sample of extracted trajectory is demonstrated in Figure 11. This is an example of dimensionality reduction. The video data of moving objects are converted into text data.

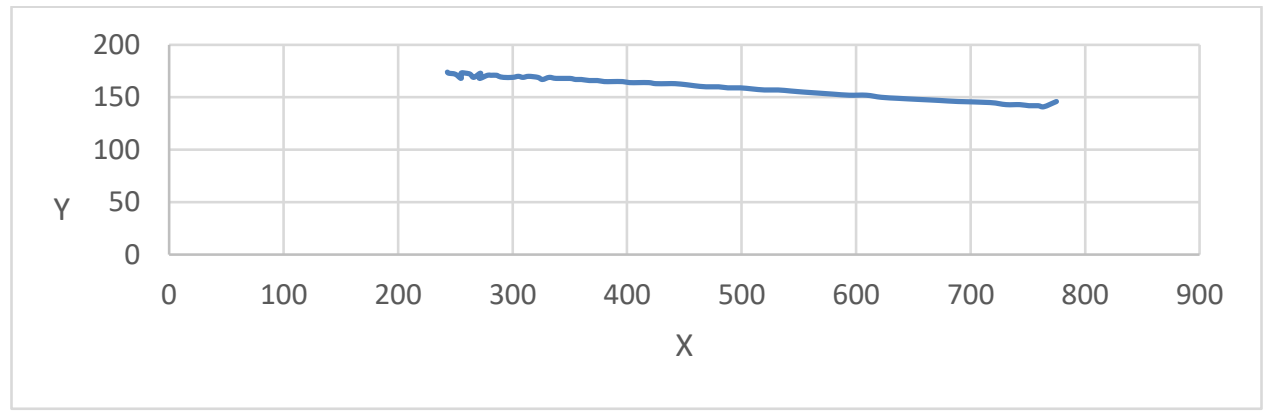

Figure 11: Example of extracted trajectory. 


\section{CONCLUSIONS}

The paper presented a model for scalable object detection, tracking and pattern recognition of moving objects that rely on dimensionality reduction with edge computing architecture. The goal of our proposed model is to build a system that processes large numbers of video files stored in a database or real time videos. This model used edge computing devices between the video camera and the cloud to reduce directionality of video data and decrease latency time and reduce network bandwidth. The figure 5 and 6 show the overall efficiency of our proposed system where network bandwidth and execution time is reduced using edge network. The proposed edge computing technique for video analytic will result in less traffic on the internet because video data are converted into text data in the edge device then text data goes to cloud for pattern recognition. This research also proposed scalable object detection and tracking of moving objects using the YOLO method that is used in edge computing device in our proposed model. The results

show that our object detection and tracking model is more accurate as shown in table 1. The novel part of this model is dimensionality reduction of video data in edge computing device and scalable object detection and tracking of moving objects. The proposed model will be helpful in real life scenarios such as traffic systems, crowd management systems, airport security systems.

In our proposed model, we implemented the prototype to measure the overall performance of our model. Future work will include using our proposed object detection and tracking model in edge device and machine learning algorithms in cloud device to determine the pattern of moving objects.

\section{REFERENCES}

Abhari, A., S. Banihashemi, and J. Li. 2017. "Object Movement Detection by Real-Time Deep Learning for Security Surveillance Camera". IEEE Conference of Computer Society, pp. 484-44.

Ahmed B, Y. Saleem, and S. Waseem. 2015. "An Implementation of Multiprotocol Label Switching Virtual Private Networks and Internet Protocol Security using Graphical Network Simulator 3 as an Educational Tool". Science international, vol. 27, no. 3, 2015.

Alam, M. S., B.V. Natesha, T.S. Ashwin, and R.M. Guddeti. 2019. "UAV Based Cost-Effictive Real-Time Abnormal Event Detection using Edge Computing”. Multimedia tools and Applications, vol 78. pp. 35119-35134.

Ali, M., A. Anjum, M. U Yaseen1, A.R. Zamani, D. Balouek-Thomert, O. Rana, and M. Parashar. 2018. "Edge Enhanced Deep Learning System for Large-Scale Video Stream Analytics". IEEE 2nd International Conference on Fog and Edge Computing, pp. 1-10.

Ananthanarayanan, G., P. Bahl, P. Bodik, K. Chintalapudi, M. Philipose, L. Ravindranath, and S. Sinha. 2017. "Real-Time Video Analytics: The Killer of App for Edge Computing". Computer, vol. 50, pp. 58-67.

Anjum, A., T Abdullah, M. Tariq, Y. Baltaci, and N. Antonopoulos. 2016. "Video Stream Analysis in Clouds: An Object Detection and Classification Framework for High Performance Video Analytics". IEEE Transactions on Cloud Computing, pp. 1-16.

Chandan, G., A. Jain, H. Jain, Mohana. 2018. Real Time Object Detection and Tracking Using Deep Learning and OpenCV. International Conference on Inventive Research in Computing Applications (ICIRCA), IEEE, pp. 1305-1308.

Farhady, H., H. Lee, and A. Nakao. 2015. "Software-Defined Networking: A survey”. Computer Networks, pp. 79-95.

Gupta, H, A. Vahid Dastjerdi, S.K. Ghosh and R. Buyya. 2017. "iFogSim: A Toolkit for Modeling and Simulation of Resource Management Techniques in the Internet of Things, Edge and Fog Computing Environments". Software: Practice and Experience, vol. 47, no. 9, pp. 1275-1296.

He, T., H. Mao, and Z. Yi. 2017. "Moving Object Recognition using Multi-View Three-dimensional Convolutional Neural Networks". Neural Computing and Applications, vol. 28, pp. 3827-3835. 
Kazi B.U.,G. Waine and V.G. da Silva. 2017."Modeling and Simulation of User Mobility and Handover in LTE and Beyond Mobile Networks using DEVS Formalism”. Spring Simulation Conference, pp.111.

Khan, W. Z., E. Ahmed, S. Hakak, I. Yagoob and A. Ahmed. 2019. "Edge Computing: A Survey". Fourth Generation Computer Systems, vol. 97, pp. 219-235, 2019.

Mahdavinejad, M.S., M. Rezvan, M. Barekatain, P. Adibi, P. Barnaghi, and A.P. Sheth. 2018. "Machine Learning for Internet of Things Data Analysis: A Survey”. Digital Communications and Networks, pp. 161-175.

Murshed M.G.S., C. Murphy, D. Hou, N. Khan, G. Ananthanarayanan, and F. Hussain. 2019. "Machine Learning at the Network Edge: A Survey". pg. 1-10, 2019.

Naha, R., A. Ray, P. Sarkar, and D. Datta. 2014. "Moving Objects Tracking in Real Time Video and Plotting Their Path of Movement". International Journal of Advanced Computer Research, vol. 4, pp. 242-250.

Nidhi, 2015. "Image Processing and Object Detection". International Journal of Applied Research, vol. 1, pp. 396-399.

Pudasaini D. and A. Abhari. 2019. "Scalable Pattern Recognition and Real Time Tracking of Moving Objects,", Spring Simulation Conference, pp. 1-11.

Redmon, j., S. Divvala, R. Girshick, and A. Farhadi, 2016. "You Only Look Once: Unified, Real-Time Object Detection". Retrieved from: https://arxiv.org/pdf/1506.02640.pdf.

Satyanarayanan, M. 2017. "The Emerging of Edge Computing. Computer, vol. 50, pp. 30-39.

Shi, W., J. Cao, Q. Zhang, Y. Li, and L. Xu. 2016. "Edge Computing: Vision and Challenges". IEEE Internet Things, vol. 3, pp. 637-646.

Singh, J. 2017. "Tracking of Moving Object using Centroid Based Prediction and Boundary Tracing Scheme". International Journal of image, graphics and signal processing, vol. 9, pp. 59-66.

Xia, X., Y. Wen, C. Foh, D. Niyato, and H. Xie. 2015. “A Survey on Software-Defined Networking”. IEEE Communications Surveys \& Tutorials, pp. 27-51.

Yadav, A., and L. B. Prasad. 2019. "IOT Devices for Control Applications: A Review". 3rd International conference on Electronics, Communication and Aerospace Technology (ICECA), pp. 473-479.

Yang, L., L. Wang, and S. Wu. 2018. "Real-time Object Recognition Algorithm Based on Deep Convolutional Neural Network". Proceedings of the $3^{\text {rd }}$ IEEE conference on cloud computing and big data analysis, pp. 331-335.

Yilmaz, A., O. Javed, and M. Shah. 20006. "Object tracking A Survey”. ACM Computing Surveys (CSUR), vol. 38, pp. 1-4.

Zhu, F., Y. Lu, N. Ying, and G. Giakos. 2017. "Fast Vehicle Detection Based on Evolving Convolutional Neural Network". Proceedings of the IEEE conference of Instrumentation and Measurement Society, pp. 1-4.

\section{AUTHOR BIOGRAPHIES}

DIPAK PUDASAINI is a PhD student in the Department of Computer Science at Ryerson University and Assistant Professor at Tribhuvan University, Nepal. He holds a M. Sc. in Computer Science from Ryerson University. His research interests include computer vision, data science, machine learning and web service selection. His email address is dpudasaini@ryerson.ca.

ABDOLREZA ABHARI is a Professor in the Department of Computer Science at Ryerson University and director of DSMP lab (http://dsmp.ryerson.ca). He holds a Ph.D. in Computer Science from Carleton University. His research interests include web social networks, data science, AI and agent systems, network simulation, and distributed systems. His email address is aabhari@ryerson.ca. 